\title{
Incidence and Outcomes of Hypernatremia in Adult Neurological Non-Brain-Dead Patients Admitted to Tertiary Care Neurologic Institute: A Retrospective Study
}

\author{
Sritam S. Jena ${ }^{1}$ Ramesh J. Venkatapura ${ }^{1}$ Rita Christopher ${ }^{2} \quad$ Dhaval Shukla $^{3}$ Dhritiman Chakrabarti ${ }^{1}$
}

${ }^{1}$ Department of Neuroanaesthesia and Neurocritical Care, National Institute of Mental Health and Neurosciences, Bengaluru, Karnataka, India

2Department of Neurochemistry, National Institute of Mental Health and Neurosciences, Bengaluru, Karnataka, India

${ }^{3}$ Department of Neurosurgery, National Institute of Mental Health and Neurosciences, Bengaluru, Karnataka, India

J Neuroanaesthesiol Crit Care 2019;6:24-29

\begin{abstract}
Address for correspondence Ramesh J. Venkatapura, MD, Department of Neuroanaesthesia and Neurocritical Care, IIIrd Floor, Neurosciences Faculty Center, National Institute of Mental Health and Neurosciences, Hosur Road, Bengaluru 560029, Karnataka, India (e-mail: drvjramesh@gmail.com).
\end{abstract}

\begin{abstract}
Keywords

- head injury

- hypernatremia

- mortality

- sodium disturbance

Background Hypernatremia is known to have high mortality and morbidity in patients with neurological disorders. However, in most studies, it is not clear whether hypernatremia associated with brain death has been excluded. Including brain-dead patients will spuriously give a very high mortality rate. Therefore, in this study, we have evaluated the mortality of hypernatremic patients after excluding the brain-dead patients on the first day of hypernatremia.

Methods All neurological patients admitted to the hospital who developed hypernatremia (serum $\mathrm{Na}>150 \mathrm{mEq} / \mathrm{L}$ ) were included in the study. Brain-dead patients and patients with Glasgow coma score (GCS) 3 on the first day of detection of hypernatremia were excluded. Demographic variables, clinical variables, and outcome variables were collected from the case files retrospectively.

Results In total, 100 patients developed hypernatremia during the study period. Among them, 14 patients were excluded because of GCS 3 or unavailability of GCS data on the day of detection of hypernatremia. There were 37 mild, 28 moderate, and 21 severe hypernatremic patients. The mortality was $32 \%, 39 \%$, and $52 \%$ in the mild, moderate, and severe hypernatremic patients, respectively. Patients with traumatic brain injury had higher mortality in comparison to all other disease conditions ( $56 \%$ vs. $29.6 \%, p<0.02$ ).

Conclusion The mortality is high even in mild cases of hypernatremia. Very high mortality (52\%) is seen in severe hypernatremic patients. Therefore, it is important to monitor, identify, and treat these patients aggressively.
\end{abstract}

\section{Introduction}

Sodium disturbances are one of the most common electrolyte disturbances seen clinically. The central nervous system (CNS) plays an important role in the regulation of sodium and water homeostasis. ${ }^{1}$ Therefore, patients suffering from CNS disorders frequently

received

December 14, 2018

accepted after revision

January 10, 2019

published online

March 6, 2019 experience sodium disturbances, which, in turn, exacerbate their neurological and general condition. These disorders frequently complicate the treatment of sodium disturbances by precipitating or worsening of neurological symptoms. Also, these patients are not only at risk for symptoms secondary to dysnatremia but also at risk from the consequences of treatment. 
Hypernatremia is seen in approximately 1 to $2 \%$ of hospitalized patients. ${ }^{2}$ Many studies have documented the incidence to be much higher in the intensive care unit (ICU)., A study has reported an incidence of $13 \%$ of hypernatremia in patients presenting to the emergency department. ${ }^{5}$ The reported mortality is also very high with severe hypernatremia. The range varies from a low of $20 \%$ to as high as $80 \%{ }^{3,6,7}$ However, it is not clear in the literature whether such a high mortality solely results from hypernatremia or studies have included all the brain-dead patients, who ultimately develop central diabetes insipidus and hypernatremia. Therefore, this study was designed to exclude all the brain-dead patients. The aim of this study was to evaluate the mortality and clinical course of hypernatremic neurological and neurosurgical patients in a tertiary care institute after excluding the brain-dead or imminent brain death viz. severely injured patients (Glasgow coma score $[\mathrm{GCS}]=3$ ) or unlikely to survive so that the attributable mortality due to hypernatremia could be determined.

\section{Methods}

Approval for this study was obtained from the institute's ethics committee. The need for informed consent was waived as data collection was retrospective. A serum sodium value of $>150$ $\mathrm{mEq} / \mathrm{L}$ was defined as hypernatremia. We included all adult (age $>18$ years) neurological and neurosurgical patients who presented with or developed hypernatremia in the hospital during December 2013 to April 2014. Hypernatremic patients were identified from the biochemistry laboratory registers, and their case records were reviewed.

\section{Exclusion Criteria}

- Patients with GCS 3 at the time of detection of hypernatremia for the first time

- Patients who were brain dead at time of detection of hypernatremia for the first time

- Patients who received treatment for correction of hypernatremia before admission to our institute

After identifying the patients, the case files were retrieved and the required information was collected. The case files contained the clinical data entered by nursing staff at the bedside, the progress notes made by physicians, prescribed drugs, and laboratory results. All included patients were followed up till their discharge/death to study the clinical course. The demographic characteristics, any comorbid disease conditions, any chronic medications, were noted. Daily vital signs, clinical features, fluid status, and any sodium correction measures were reviewed and recorded. The following laboratory data were reviewed and recorded: hemogram, serum biochemistry, and urine electrolytes and osmolality. Total durations of hospital stay, ICU stay, and mechanical ventilation were noted. GCS at discharge and in-hospital mortality were recorded.

\section{Statistical Analysis}

The mortality rate, duration of ICU stay, duration of hospital stay, and time required for correction of hypernatremia were calculated. Independent samples $t$-test/ANOVA (analysis of variance) and chi-square test/Fisher's exact test were used to test the significant difference between the groups for parametric and nonparametric data, respectively. Logistic regression analysis using Enter method was performed to identify the independent risk factors for mortality. The goodness-of-fit was assessed using "Hosmer and Lemeshow Test." A $p$-value of $<0.05$ was considered as statistically significant.

\section{Results}

A total of 4,003 patients were admitted to the hospital during the study period. Among them, 112 patients developed hypernatremia, details of which are given in - Fig. 1. Data of 86 patients were analyzed. The demographic characteristics and outcome details of the patients are given in the - Tables $1-\mathbf{3}$.

The mortality details are given in -Fig. 2. In 50 (58\%) of the patients, hypernatremia remained uncorrected. Of these 50 patients, 24 (48\%) expired. Remaining patients were either discharged with hypernatremia status or further sodium estimation was not done. In 36 (42\%) patients, hypernatremia normalized. Out of these, only $40 \%$ patients were normalized (sodium < $150 \mathrm{mEq} / \mathrm{L}$ ) by treatment. In the remaining patients, hypernatremia got corrected without treatment.

There were 31 (36\%) patients with TBI. However, the mortality in these patients was 53\%. The hypernatremia was more detrimental in patients with TBI (-Fig. 3 ).

Independent factors predicting mortality in hypernatremic patients were assessed using logistic regression. The significant independent predictors of mortality were maximum sodium value and the GCS (-Table 4).

\section{Discussion}

The definition of hypernatremia is arbitrary, but Ross and Christie in their comprehensive review suggest that most authors define hypernatremia as a serum sodium concentration $>150 \mathrm{mEq} / \mathrm{L}$ and this convention has been used in our study. ${ }^{5}$ Our division of the patients into mild, moderate, and severe hypernatremia groups is similar to the groups made by Aiyagari et al in their study. ${ }^{6}$

The mortality among hypernatremic patients varies from $20 \%$ to more than $80 \% .^{7,8}$ Our overall in-hospital mortality rate was approximately $6.8 \%$, and mortality in patients who developed hypernatremia was 34\%. The mortality rate in our study is similar to the mortality range described by different investigators.,7

Most brain-dead patients develop severe hypernatremia. ${ }^{9}$ Polyuria and hypernatremia could be induced by central diabetic insipidus resulting from insufficient blood levels of antidiuretic hormone from the posterior pituitary gland of brain-dead patients. ${ }^{10}$ At the same time, hypernatremic patients can deteriorate neurologically and they may eventually be brain dead. When we assess the mortality of hypernatremic patients, it is not clear whether hypernatremia 


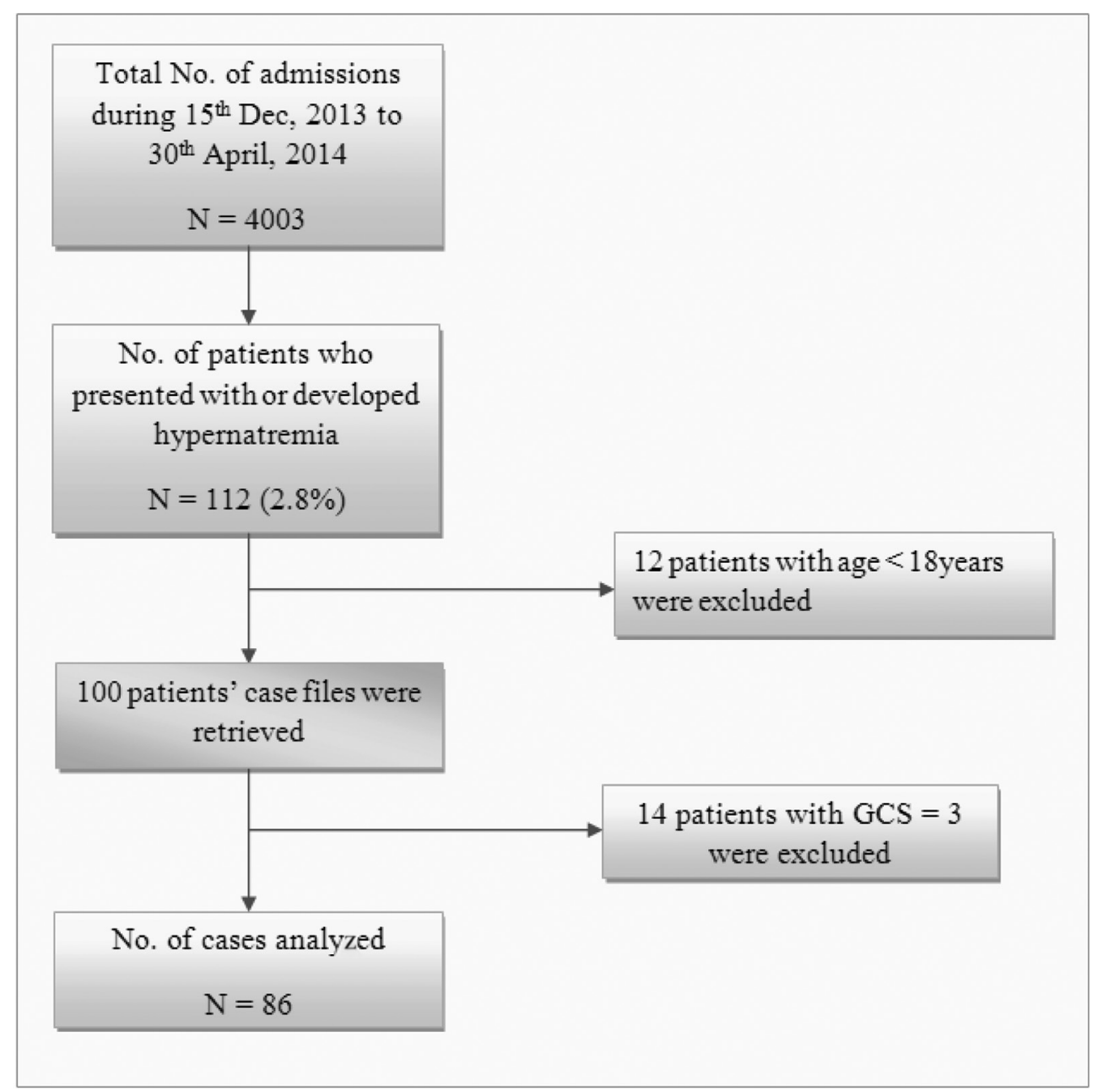

Fig. 1 Study flowchart. GCS, Glasgow coma score.

Table 1 Demographic characteristics

\begin{tabular}{|l|l|l|l|l|}
\hline \multirow{2}{*}{ Characteristics } & \multicolumn{3}{|c|}{ Hypernatremia } & $p$-Value \\
\cline { 2 - 4 } & Mild (> 150-155 mEq/L) & Moderate (> 155-160 mEq/L) & Severe (> 160 mEq/L) \\
\hline No. of patients (\%) & $37(43 \%)$ & $28(32.5 \%)$ & $21(24.5 \%)$ & $43.2 \pm 12.2$ \\
\hline $\begin{array}{l}\text { Age (y) } \\
\text { mean } \pm \text { SD) }\end{array}$ & $43.2 \pm 13.9$ & $47.4 \pm 16.7$ & $13 / 8$ & 0.7 \\
\hline Sex (M/F) & $25 / 12$ & $21 / 7$ & \\
\hline Diagnosis & $\begin{array}{l}\text { Head injury: 31 (36\%) } \\
\text { CVT/CVA: 15 (18\%) } \\
\text { CNS infection: 10 (12\%) } \\
\text { Cerebral aneurysm: 7 (8\%) } \\
\text { Brain tumor: 5 (6\%) } \\
\text { Chronic SDH: 3 (3\%) } \\
\text { Miscellaneous: 15 (17\%) (metabolic encephalopathy: 7, hydrocephalus: 4, hemorrhagic } \\
\text { leukoencephalopathy: 1, normal pressure hydrocephalus: 2, cervical spine lesion: } 1)\end{array}$ & \\
\hline
\end{tabular}

Abbreviations: CNS, central nervous system; CVA, cerebrovascular accident; CVT, cerebral venous thrombosis; F, female; M, male; SD, standard deviation; SDH, subdural hematoma.

is the cause of increased mortality or a manifestation associated with brain death. To get a clear picture, we have excluded the brain-dead patients and patients with GCS 3 while presenting with hypernatremia for the first time.
We observed that there was an incremental increase in mortality associated with the magnitude of sodium elevation as defined by sodium group. We observed mortality rate of $32.4 \%, 39.3 \%$, and $52.4 \%$ in mild, moderate, and severe 
Table 2 Details of treatment received and outcomes

\begin{tabular}{|c|c|c|c|c|}
\hline \multirow{2}{*}{\multicolumn{2}{|c|}{ Characteristics }} & \multicolumn{3}{|c|}{ Hypernatremia } \\
\hline & & Mild (151-155 mEq/L) & Moderate (> 155-160 mEq/L) & Severe (> $160 \mathrm{mEq} / \mathrm{L})$ \\
\hline \multicolumn{2}{|c|}{ Duration of hypernatremia (d; mean \pm SD) } & $1.7 \pm 1.3$ & $2.4 \pm 1.6$ & $4.4 \pm 3.3$ \\
\hline \multirow[t]{2}{*}{ Treatment received (\%) } & Fluid therapy & $7(18.9 \%)$ & $14(50 \%)$ & $11(52 \%)$ \\
\hline & Vasopressin & 0 & 0 & $1(4 \%)$ \\
\hline \multicolumn{2}{|c|}{$\begin{array}{l}\text { Prior to hypernatremia, mannitol } \\
\text { administration }\end{array}$} & \multicolumn{3}{|l|}{$\begin{array}{l}1 \text { day: } 50 \% \\
2 \text { days: } 42 \% \\
3 \text { days: } 30 \%\end{array}$} \\
\hline \multicolumn{2}{|c|}{ GCS at discharge (mean \pm SD) } & $10.7 \pm 3.2$ & $10.3 \pm 4.4$ & $10.8 \pm 3.7$ \\
\hline \multicolumn{2}{|c|}{ Incidence of hyponatremia (\%) } & $7(19 \%)$ & $3(10.7 \%)$ & $5(23.8 \%)$ \\
\hline \multicolumn{2}{|l|}{ ICU stay $(\mathrm{d}$; mean \pm SD) } & $13.8 \pm 13.14$ & $7.43 \pm 5.26$ & $13.71 \pm 7.34$ \\
\hline \multicolumn{2}{|c|}{ Hospital stay (d; mean \pm SD) } & $13.02 \pm 12.81$ & $13.74 \pm 13.25$ & $14.27 \pm 14.69$ \\
\hline \multicolumn{2}{|l|}{ Mortality } & $12(32.4 \%)$ & $11(39.3 \%)$ & $11(52.4 \%)$ \\
\hline
\end{tabular}

Abbreviations: GCS, Glasgow coma score; ICU, intensive care unit; SD, standard deviation.

Table 3 Comparison between survivors and non-survivors

\begin{tabular}{|l|l|l|l|}
\hline & $\begin{array}{l}\text { Survivors } \\
(\boldsymbol{n}=52)\end{array}$ & $\begin{array}{l}\text { Nonsurvivors } \\
(\boldsymbol{n}=\mathbf{3 4})\end{array}$ & $\boldsymbol{p}$-Value \\
\hline $\begin{array}{l}\text { Mean serum } \\
\text { creatinine value } \\
(\mathrm{mg} / \mathrm{dL})\end{array}$ & $1.39 \pm 1.02$ & $1.93 \pm 2.22$ & 0.167 \\
\hline $\begin{array}{l}\text { Mechanical ven- } \\
\text { tilation (d) (mean } \\
\pm \text { SD) }\end{array}$ & $11.7 \pm 8.7$ & $10.5 \pm 9$ & 0.75 \\
\hline $\begin{array}{l}\text { ICU stay (d) } \\
\text { (mean } \pm \text { SD) }\end{array}$ & $13.6 \pm 7.1$ & $11.2 \pm 10.6$ & 0.45 \\
\hline $\begin{array}{l}\text { Hospital stay (d) } \\
\text { (mean } \pm \text { SD) }\end{array}$ & $14.2 \pm 15.2$ & $13.8 \pm 11.1$ & 0.95 \\
\hline
\end{tabular}

Abbreviations: ICU, intensive care unit; SD, standard deviation. to that found in our study, and the mortality rates were $20 \%$, $30 \%$, and $48 \%$ in mild, moderate, and severe hypernatremic patients, respectively. In the study by $\mathrm{Li}$ et al, the mortality rates were slightly higher, that is, $18 \%, 42 \%$, and $87 \%$ in mild, moderate, and severe hypernatremic patients, respectively. Both studies included patients in TBI admitted to ICU.

Hypernatremia has multiple adverse effects on physiologic functions, which may explain its association with increased mortality. As explained earlier, hypernatremia aggravates peripheral insulin resistance; impairs hepatic gluconeogenesis, lactate clearance, and renal function; decreases left ventricular contractility; and modulates immune function. Additionally, hypernatremia is associated with various neuromuscular manifestations, such as muscle weakness and cramps. Neurological impairment is the most severe consequence of hypernatremia, and it may prolong the need for mechanical

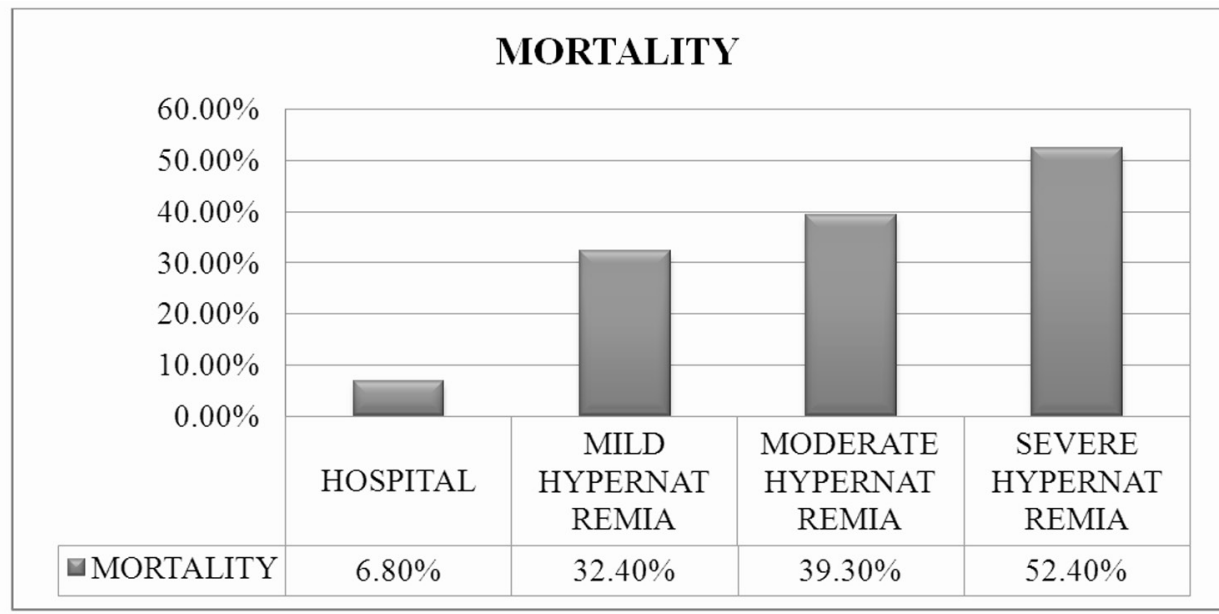

Fig. 2 Comparison of mortality rates among hypernatremia groups.

hypernatremic patients, respectively. This is similar to the findings in the studies conducted by Aiyagari et al and Li et al who also found increased risk of ICU mortality with progressive increase in hypernatremia. ${ }^{6.8}$ In the study by Aiyagari et al, the sodium range for different hypernatremic groups was similar ventilation and delay weaning. Finally, too rapid correction of chronic hypernatremia can cause cerebral edema.

We observed maximum mortality among patients with TBI who had hypernatremia during their hospital stay. The mortality among them was $52.9 \%$, which was 


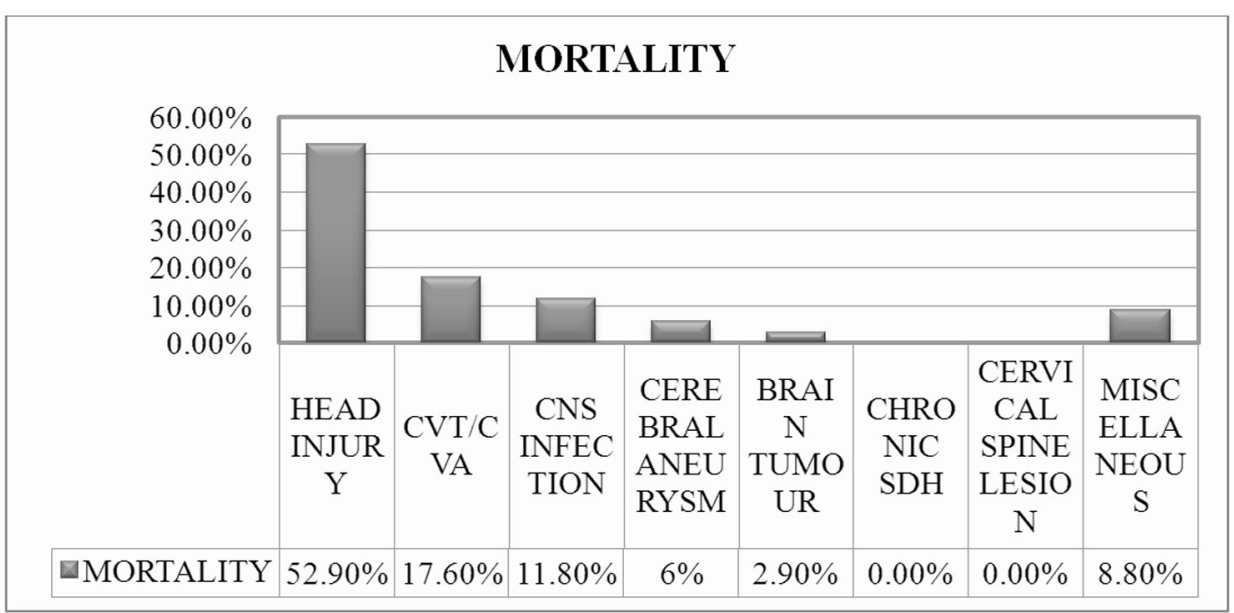

Fig. 3 Comparison of mortality rates in hypernatremia patients among various diagnostic groups. CNS, central nervous system; CVA, cerebrovascular accident; CVT, cerebral venous thrombosis.

Table 4 Factors predicting mortality in hypernatremic patients by logistic regression

\begin{tabular}{|c|c|c|c|c|c|c|}
\hline \multirow[b]{2}{*}{ Variables } & \multirow[t]{2}{*}{ B } & \multirow[t]{2}{*}{ SE } & \multirow[t]{2}{*}{ Sig. } & \multirow[t]{2}{*}{ Exp. (B) } & \multicolumn{2}{|c|}{ 95\% Cl for Exp. (B) } \\
\hline & & & & & Lower & Upper \\
\hline Sex & 1.328 & 0.867 & 0.126 & 3.774 & 0.689 & 20.664 \\
\hline Highest creatinine & 0.035 & 0.216 & 0.871 & 1.036 & 0.678 & 1.582 \\
\hline Highest sodium & 0.134 & 0.057 & 0.019 & 1.143 & 1.022 & 1.279 \\
\hline GCS & -0.373 & 0.133 & 0.005 & 0.689 & 0.530 & 0.894 \\
\hline Highest potassium & 0.112 & 0.493 & 0.821 & 1.118 & 0.425 & 2.941 \\
\hline Lowest sodium & 0.023 & 0.040 & 0.570 & 1.023 & 0.946 & 1.107 \\
\hline Highest total count & 0.000 & 0.000 & 0.089 & 1.000 & 1.000 & 1.000 \\
\hline Head injury diagnosis & -1.305 & 0.797 & 0.101 & 0.271 & 0.057 & 1.292 \\
\hline Constant & -23.610 & 11.096 & 0.033 & 0.000 & & \\
\hline
\end{tabular}

Abbreviations: $\mathrm{Cl}$, confidence interval; GCS, Glasgow coma score; SE, standard error.

similar to the finding by Li et al. ${ }^{8}$ Various factors contributing to hypernatremia in patients with TBI are impaired consciousness leading to decreased water intake, increased insensible water loss due to mechanical ventilation, increased renal loss of water due to use of hyperosmotic therapy, and/ or hypertonic sodium gain.

Several studies have found hypernatremia as an independent predictor of mortality. Most of these studies included patients with TBI and patients in general or neurological ICU population. ${ }^{11-13}$ To find the factors contributing to mortality, we did logistic regression analysis. The analysis included factors such as age, maximum potassium value $\left(\mathrm{K}^{+}\right)$, sex, maximum creatinine value, diagnosis, maximum sodium value $\left(\mathrm{Na}^{+}\right)$, GCS, total WBC count (TC), and lowest sodium value $\left(\mathrm{Na}^{+}\right)$. The maximum sodium value and GCS were independent predictors of mortality. Thus, our finding has resemblance with the findings in other studies.

There are several limitations of our study. The retrospective nature of this study meant that causality could not be determined. Our study demonstrates strong association and not necessarily causation. The severity of hypernatremia may reflect the severity of the underlying disease, and it is not possible to quantify this effect or ascribe causality.

The association between hypernatremia and adverse outcome may also be due to both the severity of underlying diseases and the direct unfavorable effects of hyperosmolality. As with all observational designs, we cannot exclude the possibility of unmeasured confounding. Finally, our study was conducted in a single center, and the generalizability of our findings to other centers is unknown.

As there is a relatively consistent finding in several studies regarding the association of hypernatremia with mortality, consideration should be given to prospective studies to evaluate the effect of early detection, prevention, and treatment of hypernatremia. Also, studies are required to identify the etiological factors leading to hypernatremia and the reason for independent association of hypernatr emia with adverse outcome. It has to be verified whether intense sodium monitoring and/or aggressive early treatment of hypernatremia results in decreased mortality. The mechanism by which an intracranial disease leads to development of hypernatremia is yet to be answered. 


\section{Conclusion}

The incidence of hypernatremia in our tertiary care neurological hospital is $2.8 \%$. Most of the mild hypernatremic patients do not receive any treatment. The mortality in hypernatremia patients is very high (32\%-52\%) even after excluding brain-dead patients. Frequent and regular monitoring of sodium along with aggressive treatment of hypernatremia may decrease the morbidity and mortality in these patients. At present, there is not much evidence to say that confidently. Future studies are required to evaluate hypernatremia and impact of its treatment.

\section{Conflict of Interest}

None declared.

\section{References}

1 Reynolds RM, Padfield PL, Seckl JR. Disorders of sodium balance. BMJ 2006;332(7543):702-705

2 Palevsky PM, Bhagrath R, Greenberg A. Hypernatremia in hospitalized patients. Ann Intern Med 1996;124(2):197-203

3 Polderman KH, Schreuder WO, Strack van Schijndel RJ, Thijs LG. Hypernatremia in the intensive care unit: an indicator of quality of care? Crit Care Med 1999;27(6):1105-1108

4 Lindner G, Funk GC, Schwarz C, et al. Hypernatremia in the critically ill is an independent risk factor for mortality. Am J Kidney Dis 2007;50(6):952-957
5 Arampatzis S, Exadaktylos A, Buhl D, Zimmermann $\mathrm{H}$, Lindner $\mathrm{G}$. Dysnatraemias in the emergency room: undetected, untreated, unknown? Wien Klin Wochenschr 2012;124(5-6):181-183

6 Aiyagari V, Deibert E, Diringer MN. Hypernatremia in the neurologic intensive care unit: how high is too high? J Crit Care 2006;21(2):163-172

7 Hoorn EJ, Betjes MG, Weigel J, Zietse R. Hypernatraemia in critically ill patients: too little water and too much salt. Nephrol Dial Transplant 2008;23(5):1562-1568

$8 \mathrm{Li} \mathrm{M}, \mathrm{Hu} \mathrm{YH}$, Chen G. Hypernatremia severity and the risk of death after traumatic brain injury. Injury 2013;44(9):1213-1218

9 Ramos HC, Lopez R. Critical care management of the braindead organ donor. Curr Opin Organ Transplant 2002;7:70-75

10 Hirschl MM, Matzner MP, Huber WO, et al. Effect of desmopressin substitution during organ procurement on early renal allograft function. Nephrol Dial Transplant 1996;11(1):173-176

11 Lindner G, Funk GC, Lassnigg A, et al. Intensive care-acquired hypernatremia after major cardiothoracic surgery is associated with increased mortality. Intensive Care Med 2010;36(10):1718-1723

12 Darmon M, Timsit J-F, Francais A, et al. Association between hypernatraemia acquired in the ICU and mortality: a cohort study. Nephrol Dial Transplant 2010;25(8):2510-2515

13 Tsipotis E, Price LL, Jaber BL, Madias NE. Hospital-associated hypernatremia spectrum and clinical outcomes in an unselected cohort. Am J Med 2018;131(1):72-82.e1 